\section{ANÁLISE DE IODATO EM SAIS DE COZINHA}

Gutto Raffyson Silva de Freitas

Humberto da Silva Junior

Francisco Régis da Silva Pereira

Robson Fernandes de Farias

Francisco Claudece Pereira*

Departamento de Química - Universidade Federal do Rio Grande do Norte - UFRN.

Caixa Postal 1524 - Campus Universitário, Lagoa Nova.

CEP. 59072-970-Natal - RN - Brasil.

(*)claudece@ufrnet.br

\section{RESUMO}

Iodato pode ser reduzido em solução tampão B-R pH 2,0 por ação da hidroxilamina. Dependendo da concentração do agente redutor é possível gerar iodo molecular ou o complexo triiodeto. Iodo apresenta um conjunto de três picos de absorção, enquanto o complexo somente dois na região do ultravioleta. Utilizando excesso de hidroxilamina é possível monitorar o triiodeto em max. $352 \mathrm{~nm}$ e max. $288 \mathrm{~nm}$. Empregando as melhores condições experimentais, curvas de calibração lineares entre 2,3×10-4 mol L-1 a 5,0x10-4 mol L-1 foram obtidas para a determinação de iodato. A metodologia foi aplicada em amostras de sais de cozinha de supermercados da cidade de Natal, RN.

Palavras-chave: iodato, triiodeto, hidroxilamina.

Palavras chaves: cromo, retenção, gibbsita, peneira molecular

\section{Introdução}


A fim de controlar muitos casos endêmicos de bócio, especialmente em habitantes de áreas montanhosas em várias regiões do mundo, a adição de iodo, ou sais derivados, na dieta humana, tem sido uma prática universal. A organização mundial da saúde (OMS) recomenda a adição de $10 \mathrm{mg}$ do elemento a $1 \mathrm{~kg}$ de sal de cozinha (o correspondente a $10 \mathrm{ppm}$ ). Em determinados países, este procedimento não é apenas opcional, mas obrigatório. Nos Estados Unidos, sais iodados apresentam $100 \mathrm{mg}$ por $\mathrm{Kg}$ do sal de cozinha, enquanto na Suiça é de somente $5 \mathrm{mg}$ por $\mathrm{Kg}$. No Brasil, o conteúdo de iodo (na forma de iodato de potássio) é controlado oficialmente em produtos comerciais, e pode ser encontrado na faixa de concentração entre 10 a $30 \mathrm{mg}$ por kilograma nos sais de cozinha, [1].

Iodo é um elemento traço essencial e necessário às funções normais da tireóide. O mesmo é empregado na síntese da tiroxina (T4) e triiodotironina (T3) pela glândula da tireóide. Uma pessoa adulta requer, diariamente, uma ingestão de cerca de 100-300 $\mu \mathrm{g}$. Quando as necessidades de suprimento de iodo não são encontradas na alimentação diária, uma série de desordens podem se desenvolver. A administração de iodo, em valores inferiores a $0,05 \mathrm{mg}$ ao dia, resulta na hipertrofia da tireóide (bócio endêmico) e, caso esta deficiência seja prolongada, pode resultar em hipotiroidismo. Por outro lado, o excesso de iodo ou iodeto também pode provocar o bócio, bem como o hipertiroidismo. Portanto, a ingestão de iodo, proveniente de comidas, drogas e águas, pode apresentar pronunciados efeitos no status da glândula da tireóide de um individuo [2].

De acordo com a OMS, cerca de 2 bilhões de pessoas, ou 35,2\% da população global, apresenta deficiência de iodo em suas necessidades diárias. $\mathrm{O}$ espectro da desordem na deficiência de iodo (DDI) no organismo humano, se estende por toda a vida e se manifesta através de aborto, anomalias congênitas, aumento na mortalidade perinatal, cretinismo endêmico, aumento da glândula da tireóide, hipotiroidismo neonatal, retardo mental, desenvolvimento físico retardado, dentre outras. A importância do iodo em países desenvolvidos tem aumentado significativamente nos últimos 10 anos $[3,4]$.

A insuficiente administração de iodo é a causa principal da DDI. Esta constatação tem levado a uma tendência global no sentido de iodizar o sal de cozinha para destinação ao público consumidor. O uso de sal iodizado é considerado o mais eficiente veículo de biodisponibilidade de comida rica em iodo. Além desta fonte, porém em menor proporção, também é possível utilizar peixes do mar, leite e determinadas forragens contendo altos teores de iodo [5]. 
Atualmente, a fortificação dos sais de cozinha com iodo tem sido prática corriqueira ao redor do mundo. Em geral, esta adição ocorre através da inserção de iodato de potássio ou sódio e, em poucos casos, com iodeto. Apesar das controvérsias sobre a adição de iodeto de sódio ou potássio nesta matriz; iodeto apresenta a propriedade de sua oxidação a iodo e severas perdas por sublimação durante os processos de industrialização e longos períodos de estocagem deste produto, além de termodinamicamente instável em águas do mar; especialmente na presença de oxigênio dissolvido. Uma vez que iodato de potássio é mais estável e, portanto, menos susceptível a reações em meio neutro ou alcalino, o mesmo é, preferencialmente, empregado nos processos de iodização do sal de cozinha [1].

Não existem dados que demonstrem alta biodisponibilidade de iodo proveniente de alimentos. Existem somente algumas evidências de que, iodo proveniente de plantas exibe baixa absorção, possivelmente devido à pobre liberação da substância durante sua digestão. Alguns estudos envolvendo a aplicação de processos de cozimento prolongado de determinadas plantas existentes no Japão, têm mostrado altos valores de excreção urinária de iodo em grupos de voluntários estudados. Esta avaliação foi realizada através da comparação de ingestão destes alimentos na forma crua e cozida [6].

Devido a presença de vários agentes redutores e oxidantes em determinados alimentos, existe uma grande possibilidade de interações do iodo e iodato com estas espécies redox. Contudo, não há estudos científicos que avaliem a interação do iodato em uma matriz alimentar, ou in vivo, durante os processos de digestão ou absorção do alimento. Como exemplo clássico, cita-se o caso de alguns sais de cozinha que, além de serem fortificados com iodato, também recebem porções generosas de sais de ferro para combater anemias. Ocorre que, tanto iodo como iodato, pode reagir com ferro, ou impurezas nesta matriz, provocando perdas do produto iodo [7].

Durante a manufatura do sal de cozinha é adicionado uma quantidade de iodo que pode varia entre 20 a $40 \mathrm{mg}$ por kilograma do sal. Este valor corresponde a cerca de 20 a 40 ppm de iodo, capaz de fornecer $150 \mu \mathrm{g}$ de iodo por dia a um indivíduo. Esta adição ocorre na forma de iodato de sódio ou potássio. Nestas circunstâncias, os níveis de excreção urinária de iodo situamse entre 100 a $200 \mu \mathrm{g} \mathrm{L}^{-1}$ [8].

No ano de 1991, o comitê que avalia aditivos em alimentos da OMS, considerou que iodato de potássio exibe um longo histórico no seu emprego como fortificante, sem apresentar efeitos 
adversos à saúde humana. Em adição, nenhuma evidência científica indica perigo toxicológico por ingestão deste sal acima do nível de tolerabilidade (1 $\mathrm{mg}$ de iodo). Dados deste estudo mostram que, de 38 países da Europa e adjacências, 5 permitem o uso somente de iodato; outros 20, tanto aceitam iodato como iodeto e 13 somente iodeto. Em função de recomendações de autoridades da saúde, o uso de iodato prevalece em detrimento ao iodeto. O mesmo é largamente empregado como fonte de iodo na dieta alimentar das pessoas [9].

A toxicidade e outros perigos do iodato não são reportados por agências regulatórias. A união européia considera iodato não tóxico, enquanto os Estados Unidos não efetuam qualquer registro sobre os cuidados em relação ao mesmo. Como aditivo em alimentos é geralmente reconhecido como seguro, e pode ser adicionado em farinhas até 0,0075\% (75ppm). Baixos limites de iodato também são aceitos em suplementos minerais para animais [9].

Não existem dados sobre a genotoxicidade para iodato. Raros trabalhos têm mostrado que ele não induz mutações em Salmonella typhimurium ou não aumenta a freqüência de nucleação em células de medula de ratos contaminados com Drosophila melanogaster. Outros registros têm reportado a atividade anti-mutagênica de iodato, em relação a aflatoxina $\mathrm{B} 1$, através de testes envolvendo a bactéria Salmonella. Não existem dados sobre a carcinogenicidade para iodado [9]. Normalmente as medidas quantitativas para iodato, em amostras alimentares, são realizadas iodometricamente, via titulação com tiossulfato, após sua liberação em reações redox em meio ácido. Distintos protocolos têm sido elaborados para a análise de iodato, tais como, espectrofotometria [1, 10-21]; métodos em FIA [22-27]; cromatografia iônica [28, 29]; cromatografia gasosa [2, 30]; cromatografia liquida [31]; eletroquímica [23, 24, 32-37] e quimiluminescência [38].

Assim, e considerando a importância de métodos analíticos capazes de monitorar iodato, o presente trabalho descreve as melhores condições experimentais para a sua determinação em sais de cozinha, após reação com hidroxilamina, e monitoramento do produto empregando técnica espectrofotométrica.

\section{Experimental}

\section{Equipamentos}

Espectrofotômetro UV-VIS 1650 PC da marca Shimadzu. Todos os experimentos foram conduzidos com cubetas de quartzo com $1 \mathrm{~cm}$ de caminho ótico. Para a preparação das soluções 
de trabalho foi empregada uma balança analítica da marca Marte Mict. Todos os valores de $\mathrm{pH}$ das soluções utilizadas foram ajustados com um pHmetro da marca Marconi PA200, modelo RS232. Antes de seu uso, o eletrodo combinando de vidro foi previamente calibrado com as soluções fornecidas pelo fabricante.

\section{Reagentes}

Iodato de potássio (Merck), iodeto de potássio (Merck), cloridrato de hidroxilamina $\left(\mathrm{NH}_{2} \mathrm{OH} . \mathrm{HCl}\right)$ (Vetec), iodo, ácido acético (Vtec), ácido fosfórico (Merck), ácido bórico (Merck), ácido nítrico (Merck), hidróxido de sódio (Merck), cobre metálico. Todos os reagentes apresentavam grau analítico.

\section{Procedimentos experimentais}

Soluções tampão Britton e Robinson (B-R) foram obtidas através da mistura dos ácidos acético, fosfórico e bórico. $\mathrm{O} \mathrm{pH}$ das soluções, no intervalo de 2 a 12, foi ajustado pela adição de hidróxido de sódio. Soluções dos sais utilizados foram obtidas através da sua dissolução diretamente em água. Todas as soluções foram preparadas utilizando água bidestilada.

Solução de triiodeto foi obtida pela dissolução de iodo molecular $\left(\mathrm{I}_{2}\right)$ em excesso de solução de iodeto de potássio. Solução de iodo $\left(\mathrm{I}_{2}\right)$ foi gerada através da dissolução do composto em água (solubilidade do $\mathrm{I}_{2}=0,030 \mathrm{~g}$ em $100 \mathrm{ml}$ de água a $25^{\circ} \mathrm{C}$, correspondente a $\mathrm{M}\left(\mathrm{I}_{2}\right)=1,18 \times 10^{-3} \mathrm{~mol}$ $\left.\mathrm{L}^{-1}\right)$.

Testes envolvendo o gás NO (óxido nítrico) foram realizados após a geração de NO, através da dissolução de cobre metálico em ácido nítrico concentrado. O gás foi borbulhado em água, em soluções de iodo molecular e em soluções de triiodeto. Todas os sistemas encontravam-se tamponados em B-R pH 2,0.

As amostras analisadas foram os sais Marlin, Nevado e Leste. Todos comprados nos supermercados da cidade de Natal-RN. Uma massa de 32g de cada marca foi dissolvida em água para um volume final de $100 \mathrm{~mL}$, e analisadas de acordo com as melhores condições experimentais inferidas no presente trabalho.

\section{Resultados e discussões}


$\mathrm{O}$ comportamento espectrofotométrico $\mathrm{do} \mathrm{IO}_{3}^{-}$foi inicialmente investigado para valores de concentração de $2 \times 10^{-6} \mathrm{~mol} \mathrm{~L}^{-1}$ a $6 \times 10^{-6} \mathrm{~mol} \mathrm{~L}^{-1}$ em solução tampão B-R em valores de $\mathrm{pH}$ de 2 a 12,0. Para estas condições experimentais, iodato não exibe nenhum máximo de absorção que possibilite sua análise espectrofotométrica em toda a região de comprimento de onda de 200 a $800 \mathrm{~nm}$. Estes resultados estão de acordo com a literatura que reporta que iodato não pode ser analisado diretamente através desta técnica [39].

Considerando que $\mathrm{IO}_{3}{ }^{-}$não apresenta um sinal analítico que permita seu monitoramento e, uma vez que o mesmo é um forte agente oxidante (Eo= 1,194 V em meio ácido) [40], avaliou-se, a seguir, a possibilidade do emprego de um agente redutor capaz de interagir com iodato, com a finalidade de desenvolver um método derivatizado que permita o seu monitoramento espectrofotométrico.

De acordo com a literatura [41, 42], hidroxilamina é um forte agente redutor capaz de reduzir várias espécies químicas. Dentro deste contexto, soluções de $\mathrm{NH}_{2} \mathrm{OH}$, em concentrações de $4 \times 10^{-3} \mathrm{~mol} \mathrm{~L}^{-1}$ e $8 \times 10^{-3} \mathrm{~mol} \mathrm{~L}^{-1}$ foram submetidas a uma avaliação espectrofotométrica em solução tampão B-R pH 2 a 4. Nenhum sinal foi observado para estas condições experimentais. Em seguida, foram misturadas alíquotas de soluções de $\mathrm{IO}_{3}^{-}$, em concentração de $8 \times 10^{-4}$ mol L ${ }^{-1}$, e $\mathrm{NH}_{2} \mathrm{OH} . \mathrm{HCl}$ em valores crescentes de concentração entre $8 \times 10^{-4} \mathrm{~mol} \mathrm{~L}^{-1}$ e $2 \times 10^{-3} \mathrm{~mol}$ $\mathrm{L}^{-1}$ em solução tampão B-R pH 2,0. Os resultados desta avaliação preliminar são mostrados na Figura 1.

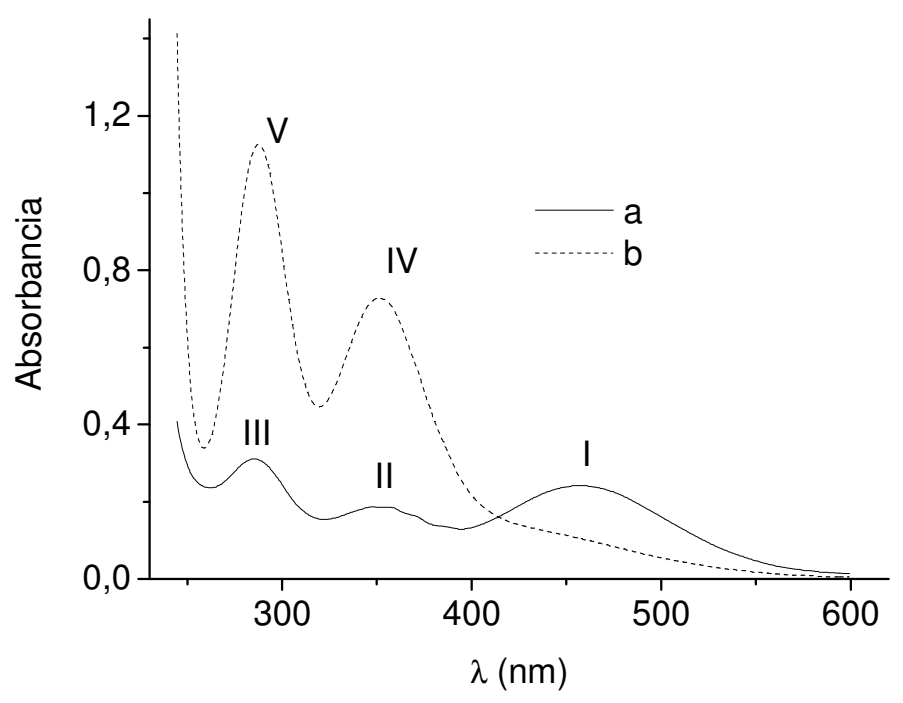


Figura 1: Espectros de absorção molecular obtidos da mistura de $8 \times 10^{-4} \mathrm{~mol} \mathrm{~L}^{-1}$ de $\mathrm{IO}_{3}{ }^{-}$e adições de $\mathrm{NH}_{2} \mathrm{OH} . \mathrm{HCl}$ em $8 \times 10^{-4}$ mol L ${ }^{-1}$ (curva "a") e $2 \times 10^{-3} \mathrm{~mol} \mathrm{~L}^{-1}$ (curva "b") em solução tampão B-R pH 2,0.

A referida figura mostra que, o produto da reação entre $\mathrm{IO}_{3}{ }^{-}$e $\mathrm{NH}_{2} \mathrm{OH}$, ambos em concentração de $8 \times 10^{-4} \mathrm{~mol} \mathrm{~L}^{-1}$, (curva "a") apresenta um conjunto de picos em $\lambda_{\max } 460 \mathrm{~nm}$ (pico I), $\lambda_{\text {max. }} 352$ $\mathrm{nm}$ (pico II) e $\lambda_{\max } 285 \mathrm{~nm}$ (pico III). Por outro lado, a curva espectral correspondente a mistura de $\mathrm{IO}_{3}{ }^{-}$em concentração de $8 \times 10^{-4} \mathrm{~mol} \mathrm{~L}^{-1}$ e $\mathrm{NH}_{2} \mathrm{OH}$ em concentração de $2 \times 10^{-3} \mathrm{~mol} \mathrm{~L}^{-1}$ (curva "b"), exibe um par de picos em valores de $\lambda_{\max } 352 \mathrm{~nm}$ (pico IV) e $\lambda_{\max .} 289 \mathrm{~nm}$ (pico V).

Segundo a literatura [43], os parâmetros extraídos dos resultados acima apresentados são característicos de sistema redox, cujo produto pode ser iodo $\left(\mathrm{I}_{2}\right)$ (curva "a") e triiodeto (curva "b").

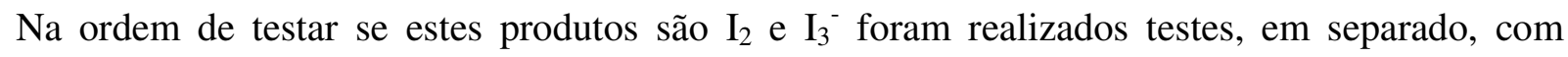
soluções de $1,0 \times 10^{-3} \mathrm{~mol} \mathrm{~L}^{-1}$ de iodo e $1,0 \times 10^{-3} \mathrm{~mol} \mathrm{~L}^{-1}$ de iodo (na presença de excesso de iodeto). A investigação foi conduzida em solução tampão B-R pH 2,0. Os resultados são mostrados na Figura 2.

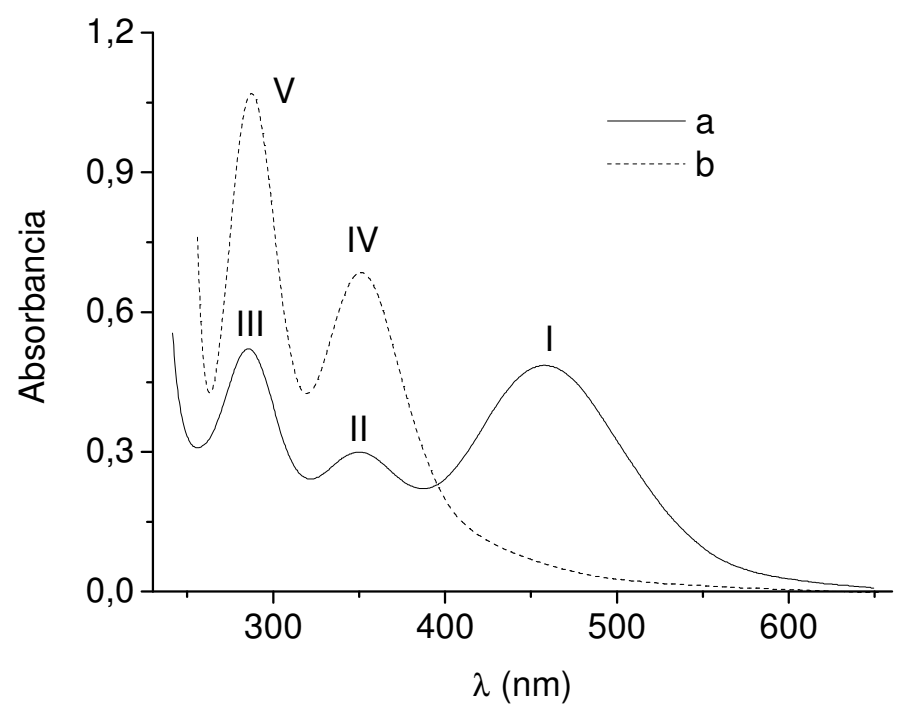


Figura 2: Espectros UV-Vis obtidos em B-R pH 2,0. Curva "a": 1,0x $10^{-3} \mathrm{~mol} \mathrm{~L}^{-1}$ de iodo e curva "b": triiodeto $\left(1,0 \times 10^{-3} \mathrm{~mol} \mathrm{~L}^{-1}\right.$ de iodo em excesso de iodeto).

O espectro obtido para iodo (curva "a") é caracterizado por um conjunto de três picos centrados em $\lambda_{\max .} 460 \mathrm{~nm}$ (pico I), $\lambda_{\max } 350 \mathrm{~nm}$ (pico II) e $\lambda_{\max } 285 \mathrm{~nm}$ (pico III). Estes achados corroboram com a literatura que estabelece este comportamento espectrofotométrico como sendo para a substância molecular iodo [43]. Por sua vez, os resultados do ensaio para triiodeto (curva "b") indica uma curva que apresenta duas bandas de absorção centradas em $\lambda_{\max }$. $352 \mathrm{~nm}$ (pico IV) e $\lambda_{\max } 287 \mathrm{~nm}$ (pico V). Este comportamento está associado ao complexo triiodeto [43]. Outros experimentos foram realizados mantendo estas condições constantes e variando o pH da solução tampão no intervalo entre $2,0 \leq \mathrm{pH} \leq 5,0$. Os resultados apresentaram o mesmo perfil.

Diante destes dados é possível depreender que hidroxilamina $\left(\mathrm{NH}_{2} \mathrm{OH}\right)$ é capaz de reduzir iodato $\left(\mathrm{IO}_{3}^{-}\right)$a iodo $\left(\mathrm{I}_{2}\right)$ e, este produto, sofrer novo processo sendo convertido a triiodeto $\left(\mathrm{I}_{3}{ }^{-}\right)$.

A expressão química para a conversão do iodato a iodo pode ser representado pela equação (1):

$6 \mathrm{IO}_{3}^{-}+6 \mathrm{H}^{+}+10 \mathrm{NH}_{2} \mathrm{OH} \rightarrow 3 \mathrm{I}_{2}+18 \mathrm{H}_{2} \mathrm{O}+10 \mathrm{NO}$

Assim, e considerando os resultados mostrados na Figura 1 (curva a), onde as concentrações de $\mathrm{IO}_{3}{ }^{-}$e $\mathrm{NH}_{2} \mathrm{OH} . \mathrm{HCl}$ são iguais em $8 \times 10^{-4} \mathrm{~mol} \mathrm{~L}^{-1}$ é possível postular que o produto, expresso na equação 1, é o iodo, uma vez que as concentração são iguais e não sobra reagente para converter o iodo a seu complexo. Para a conversão estequiométrica $\left(\mathrm{IO}_{3}{ }^{-} \rightarrow \mathrm{I}_{2}\right)$ a razão molar é de $5 / 3$ de $\mathrm{NH}_{2} \mathrm{OH}$ em relação ao $\mathrm{IO}_{3}{ }^{-}$. Neste caso, conclui-se que toda a $\mathrm{NH}_{2} \mathrm{OH}$ foi consumida no processo de conversão do iodato a iodo.

A expressão química que poderia expressar a conversão do iodo a seu complexo pode ser representada pela relação 2 .

$2 \mathrm{NH}_{2} \mathrm{OH}+9 \mathrm{I}_{2} \rightarrow 2 \mathrm{NO}+6 \mathrm{H}^{+}+6 \mathrm{I}_{3}^{-}$

Uma avaliação nos resultados já apresentados, é possível constatar que o produto da reação final (curva "b" da Figura 1) é o complexo triiodeto. Neste caso a concentração de $\mathrm{NH}_{2} \mathrm{OH}$ é 2,5 vezes maior $\left(2 \times 10^{-3} \mathrm{~mol} \mathrm{~L}^{-1}\right)$ em relação ao iodato (mantida constante em $\left.8 \times 10^{-4} \mathrm{~mol} \mathrm{~L}^{-1}\right)$. Assim, existe um excesso de $\mathrm{NH}_{2} \mathrm{OH}$, proveniente da reação 1, capaz de provocar a formação do complexo triiodeto (equação 2), uma vez que a quantidade estequiométrica para o primeiro 
processo requer somente uma relação de $(1,666 \ldots)$ de $\mathrm{NH}_{2} \mathrm{OH}$ para cada iodato. $\mathrm{O}$ excedente dos 2,5 é a quantidade de matéria necessária para provocar o segundo evento químico.

A seguir, efetuou-se uma investigação sobre a influência no aumento na concentração de iodato, na faixa entre $8 \times 10^{-4} \mathrm{~mol} \mathrm{~L}^{-1}$ a $5 \times 10^{-3} \mathrm{~mol} \mathrm{~L}^{-1}$, sobre uma concentração fixa de hidroxilamina $\left(3 \times 10^{-3} \mathrm{~mol} \mathrm{~L}^{-1}\right)$ em solução tampão B-R pH 2,0. Os resultados são mostrados na Figura 3.
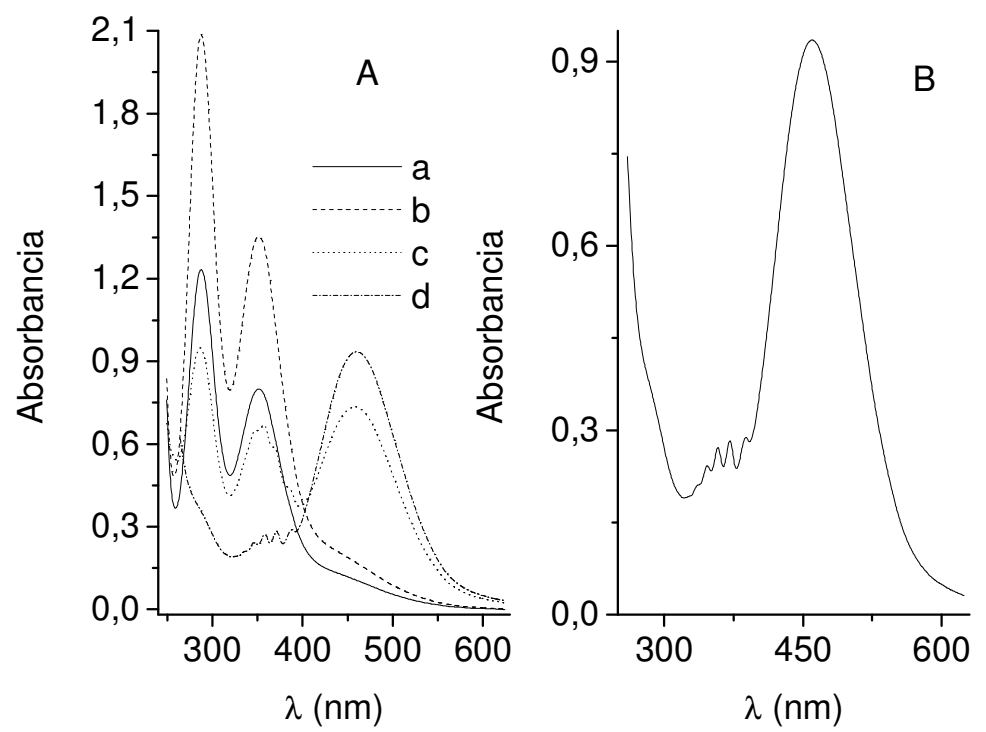

Figura 3A: Espectros UV-Vis obtidos em solução tampão B-R pH 2,0 para as misturas de $3 \times 10^{-3}$ mol L ${ }^{-1}$ de $\mathrm{NH}_{2} \mathrm{OH} . \mathrm{HCl}$ e adições de $\mathrm{IO}_{3}{ }^{-}$. Curvas (a) $8 \times 10^{-4} \mathrm{~mol} \mathrm{~L}^{-1}$, (b) $=1 \times 10^{-3} \mathrm{~mol} \mathrm{~L}^{-1}$, (c) $=$ $2 \times 10^{-3} \mathrm{~mol} \mathrm{~L}^{-1} \mathrm{e}(\mathrm{d})=5 \times 10^{-3} \mathrm{~mol} \mathrm{~L}^{-1}{\mathrm{de} \mathrm{IO}_{3}}^{-}$. Figura 3B: Ampliação da curva "d" da Figura 3A.

Os resultados evidenciam que, quando a concentração do redutor $\left(\mathrm{NH}_{2} \mathrm{OH}\right)$ é majoritária, todo o iodato adicionado, é convertido, instantaneamente, a triiodeto. Esta relação pode ser vislumbrada no espectro (a), onde a concentração da $\mathrm{NH}_{2} \mathrm{OH}$ é $3 \times 10^{-3} \mathrm{~mol} \mathrm{~L}^{-1}$ e $\mathrm{IO}_{3}^{-} 8 \times 10^{-4} \mathrm{~mol} \mathrm{~L}^{-1}$. Mantendo constante a concentração da $\mathrm{NH}_{2} \mathrm{OH}$ e aumentando a concentração de $\mathrm{IO}_{3}{ }^{-}$para $1 \times 10^{-3}$ mol L ${ }^{-1}$ (curva b), novamente observa-se que a intensidade do sinal do $\mathrm{I}_{3}{ }^{-}$é aumentado. Neste 
contexto é possível postular uma equação química, para a direta conversão do $\mathrm{IO}_{3}{ }^{-}$a $\mathrm{I}_{2}$, representado pela expressão:

$9 \mathrm{IO}_{3}^{-}+6 \mathrm{H}^{+}+16 \mathrm{NH}_{2} \mathrm{OH} \rightarrow 3 \mathrm{I}_{3}^{-}+27 \mathrm{H}_{2} \mathrm{O}+16 \mathrm{NO}$

Considerando que a relação de hidroxilamina e iodato, para a conversão direta $\mathrm{de}_{\mathrm{IO}_{3}}{ }^{-}$a $\mathrm{I}_{3}{ }^{-}$é $(1,77 \ldots .$.$) pode-se perceber que as razões de concentração de hidroxilamina/iodato nos$ resultados mostrados nas curvas (a e b) da Figura 3A são 3,75 e 3,0, respectivamente. Assim, existe $\mathrm{NH}_{2} \mathrm{OH}$ suficiente para a conversão do iodato a triiodeto, diretamente.

Todavia, quando a relação molar entre hidroxilamina e iodato é diminuída a valores abaixo de $(1,77 \ldots .$.$) , é possível constatar outros processos químicos no meio reacional. Por exemplo, a curva$ (c) da Figura $3 \mathrm{~A}$, corresponde a mistura de $3 \times 10^{-3} \mathrm{~mol} \mathrm{~L}^{-1}$ de $\mathrm{NH}_{2} \mathrm{OH}$ e $2 \times 10^{-3} \mathrm{~mol} \mathrm{~L}^{-1}$ de $\mathrm{IO}_{3}$. Nestas condições, a razão molar entre hidroxilamina e iodato assume valor de 1,5; valor esse, menor que o necessário para a total conversão do $\mathrm{IO}_{3}{ }^{-}$a $\mathrm{I}_{3}{ }^{-}$. Neste caso, o sinal espectrofotométrico do triiodeto é atenuado, enquanto observa-se a formação do iodo molecular $\left(\mathrm{I}_{2}\right)$. De acordo com a equação 1 , a razão molar entre hidroxilama e iodato, para a conversão do agente oxidante a iodo molecular, seria $(1,666 \ldots .$.$) . Como esse valor é muito próximo da relação$ de 1,5; a curva espectral mostrada em (c) assume as características do produto iodo.

Quando se incrementa a concentração de iodato no meio reacional, para valor de concentração de $5 \times 10^{-3} \mathrm{~mol} \mathrm{~L}^{-1}$ ou superior (Figura 3B), observa-se a geração de outros produtos. $\mathrm{O}$ correspondente espectro mostra uma intensa banda de absorção em $\lambda_{\max } 460 \mathrm{~nm}$, seguido de pequenos eventos ópticos em $\lambda$ de $387 \mathrm{~nm}, 371 \mathrm{~nm}, 358 \mathrm{~nm}$ e $346 \mathrm{~nm}$.

Segundo a literatura [44], iodo molecular $\left(\mathrm{I}_{2}\right)$ reage com óxido nítrico $(\mathrm{NO})$, formando iodeto de nitrosila, segundo a equação 4.

$\mathrm{I}_{2}+2 \mathrm{NO} \rightarrow 2 \mathrm{NOI}$

$\mathrm{Na}$ ordem de confirmar esta possibilidade, NO foi gerado a partir da dissolução de cobre metálico e ácido nítrico concentrado, de acordo com a equação 5.

$3 \mathrm{Cu}+8 \mathrm{HNO}_{3} \rightarrow 3 \mathrm{Cu}^{+2}+6 \mathrm{NO}_{3}^{-}+4 \mathrm{H}_{2} \mathrm{O}+2 \mathrm{NO}$

O óxido nítrico obtido foi borbulhado, em distintos tempos de borbulhamento, em solução saturada de iodo no sistema tampão B-R pH 2,0. Os resultados são mostrados na Figura 4A. 


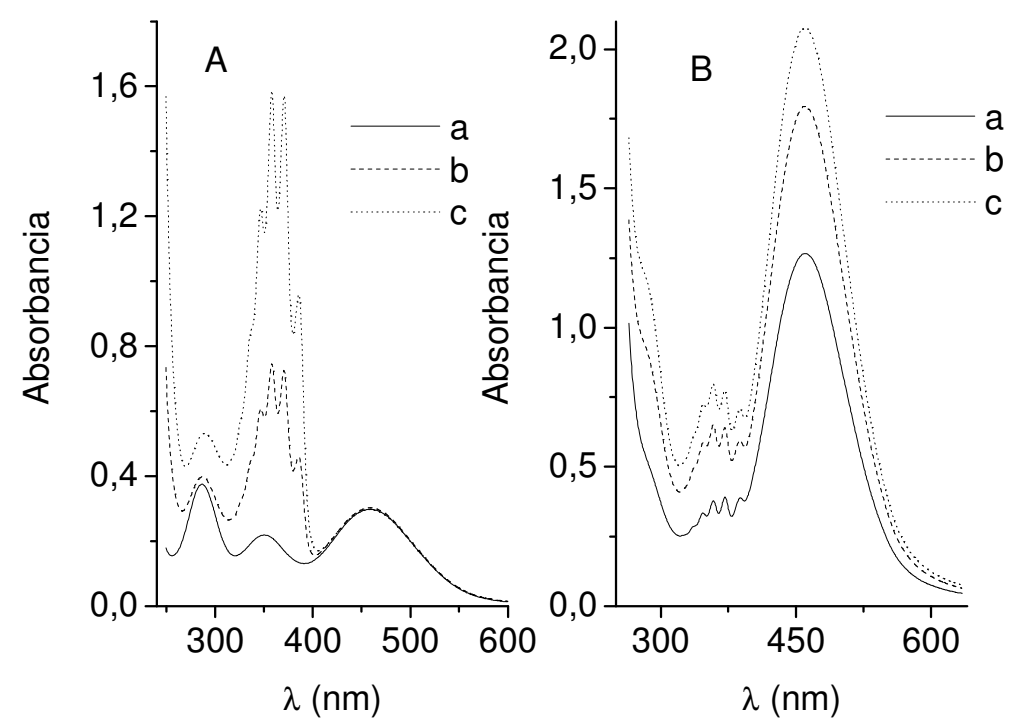

Figura 4A: Espectros UV-Vis obtidos de solução saturada de $\mathrm{I}_{2}$ em tampão B-R pH 2,0

(curva a), seguido de borbulhamento de $\mathrm{NO}$ em tempos de: 60segundos (curva b) e 180segundos (curva c). Figura 4B: Espectros UV-Vis obtidos de solução de $1 \times 10^{-2}$ mol L ${ }^{-1}$ de $\mathrm{IO}_{3}{ }^{-}$em tampão B-R pH 2,0, seguido da adição de $\mathrm{NH}_{2} \mathrm{OH}$. Curvas: $(\mathrm{a})=5 \times 10^{-3} \mathrm{~mol} \mathrm{~L}^{-1},(\mathrm{~b})=8 \times 10^{-3} \mathrm{~mol} \mathrm{~L}^{-1} \mathrm{e}(\mathrm{c})=1 \times 10^{-2} \mathrm{~mol} \mathrm{~L}^{-1}$.

A curva "a" da Figura 4A mostra o espectro UV-Vis da solução saturada de iodo. As características são similares àquelas mostradas na Figura 2. Em seguida, efetuou-se o borbulhamento de NO, obtendo-se os resultados mostrados nas curvas "b e c" da referida figura. É possível observar a formação de um produto entre o $\mathrm{NO}$ e $\mathrm{I}_{2}$. Esta nova substância apresenta um conjunto de novos picos de absorção em $\lambda_{\max } 386 \mathrm{~nm}, 371 \mathrm{~nm}, 358 \mathrm{~nm}$ e $346 \mathrm{~nm}$, além daqueles previamente conhecidos. O sinal deste composto aumenta em função do tempo de exposição do NO à solução de iodo (curva c na Figura 4A).

A seguir, uma nova solução de $1 \times 10^{-2} \mathrm{~mol} \mathrm{~L}^{-1}$ de $\mathrm{IO}_{3}{ }^{-}$em tampão B-R pH 2,0, foi submetida a adição de $\mathrm{NH}_{2} \mathrm{OH}$ na faixa de concentração entre $5 \times 10^{-3} \mathrm{~mol} \mathrm{~L}^{-1}$ a $1 \times 10^{-2} \mathrm{~mol} \mathrm{~L}^{-1}$. Os resultados são mostrados na Figura 4B. A análise desta figura permite depreender que, a adição de alíquota de $5 \times 10^{-3}$ mol L $\mathrm{L}^{-1}$ de $\mathrm{NH}_{2} \mathrm{OH}$ (curva a) é capaz de gerar o produto iodo. Contudo, para esta condição, não existe $\mathrm{NH}_{2} \mathrm{OH}$ suficiente para a conversão de $\mathrm{I}_{2}$ ao seu complexo [ $\mathrm{I}_{3}{ }^{-}$]. Todavia, uma fração do iodo produzido, reage com NO formando o iodeto de nitrosila, segundo a equação 
4, e os sinais deste produto são visíveis nos $\lambda_{\max }$ de $387 \mathrm{~nm}, 371 \mathrm{~nm}, 358 \mathrm{~nm}$ e $346 \mathrm{~nm}$. Adições posteriores de $\mathrm{NH}_{2} \mathrm{OH}$ (curvas b e c) promovem a formação de mais NOI e os sinais aumentam em função dos incrementos de $\mathrm{NH}_{2} \mathrm{OH}$. Também é possível observar que o sinal do iodo, em $\lambda_{\text {max. }} 460 \mathrm{~nm}$ aumenta a medida que novas alíquotas de $\mathrm{NH}_{2} \mathrm{OH}$ são adicionadas ao meio reacional. A comparação das intensidades dos sinais do produto (NOI), gerado através da reação da $\mathrm{NH}_{2} \mathrm{OH}$ com $\mathrm{IO}_{3}{ }^{-}$, mostra que a redução do $\mathrm{HNO}_{3}$ com $\mathrm{Cu}$ (equação 5), é capaz de gerar uma quantidade maior de $\mathrm{NO}$ e, sua subseqüente interação com $\mathrm{I}_{2}$.

A seguir efetuo-se uma investigação sobre a influência na variação de $\mathrm{pH}$, em sistema tamponado B-R, sobre os sinais espectrofotométricos da mistura de $5 \times 10^{-4} \mathrm{~mol} \mathrm{~L}^{-1}$ de iodato, e hidroxilamina em valores de concentração entre $4 \times 10^{-4} \mathrm{~mol} \mathrm{~L}^{-1}$ a $3,0 \times 10^{-3} \mathrm{~mol} \mathrm{~L}^{-1}$. Para estas condições experimentais, somente em pH 2,0, foram observados resultados. A Figura 5 mostra os dados deste experimento.
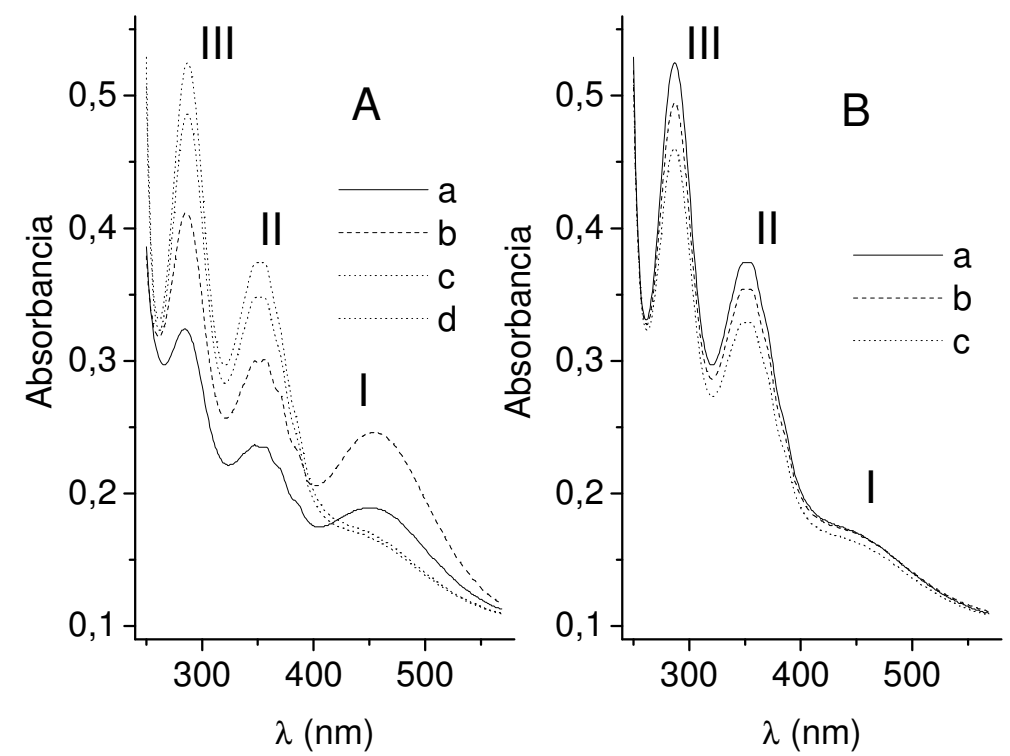

Figura 5 (A): Espectros de absorção UV-Vis da mistura em B-R pH 2,0 de 5,0x10 ${ }^{-4} \mathrm{~mol} \mathrm{~L}^{-1}$ de iodato e hidroxilamina em valores de concentração de: curva (a) $4,0 \times 10^{-4} \mathrm{~mol} \mathrm{~L}^{-1}$, (b) $6,0 \times 10^{-4} \mathrm{~mol} \mathrm{~L}^{-1}$, (c) $1,0 \times 10^{-3} \mathrm{~mol} \mathrm{~L}^{-1} \mathrm{e}$ (d) $1,5 \times 10^{-3} \mathrm{~mol} \mathrm{~L}^{-1}$. (B): Espectros de absorção UV-Vis da mistura em B-R pH 2,0 de 5,0x $10^{-4} \mathrm{~mol} \mathrm{~L}^{-1}$ de iodato e hidroxilamina em 
valores de concentração de: curva (a) $2,0 \times 10^{-3} \mathrm{~mol} \mathrm{~L}^{-1}$, (b) $2,5 \times 10^{-3} \mathrm{~mol} \mathrm{~L}^{-1} \mathrm{e}$ (c) $3,0 \times 10^{-3} \mathrm{~mol} \mathrm{~L}^{-1}$.

Os resultados mostrados na Figura 5A indicam que, quando as concentrações de $\mathrm{IO}_{3}{ }^{-}$e $\mathrm{NH}_{2} \mathrm{OH}$ estão na proporção de 5:4 e 5:6 (curvas "a" e "b"), respectivamente, os espectros acusam os sinais do produto iodo (picos I, II e III). A medida que a concentração de agente redutor aumenta, o iodo é convertido ao complexo triiodeto (picos II e III) e, os sinais aumentam até a relação de iodato e hidroxilamina na proporção de 1:2 e 1:3 (curvas "c" e "d"), respectivamente. Estes achados estão em consonância com discussão prévia.

Todavia, quando a relação entre iodato e hidroxilamina é inferior a 1:3 os sinais do complexo (picos II e III) tendem a sofrer rebaixamento, conforme mostram as curvas da Figura 5B, onde a concentração de iodato é $5,0 \times 10^{-4} \mathrm{~mol} \mathrm{~L} \mathrm{~L}^{-1} \mathrm{e}$ da hidroxilamina $2,0 \times 10^{-3} \mathrm{~mol} \mathrm{~L}^{-1}$ (curva "a"), $2,5 \times 10^{-3} \mathrm{~mol} \mathrm{~L}^{-1}$ (curva “b”) e $3,0 \times 10^{-3} \mathrm{~mol} \mathrm{~L}^{-1}$ (curva “c"), respectivamente.

Estes resultados mostram que é importante a relação molar entre os reagentes empregados para a análise do produto desejado (o complexo triiodeto). Assim, escolheu-se a relação de 1:3 entre iodato e hidroxilamina, respectivamente, para estudos futuros.

Uma curva de calibração para iodato foi obtida, em solução tampão B-R pH 2,0, através de misturas iodato e hidroxilamina na proporção 1:3. Os resultados são mostrados na Figura 6.
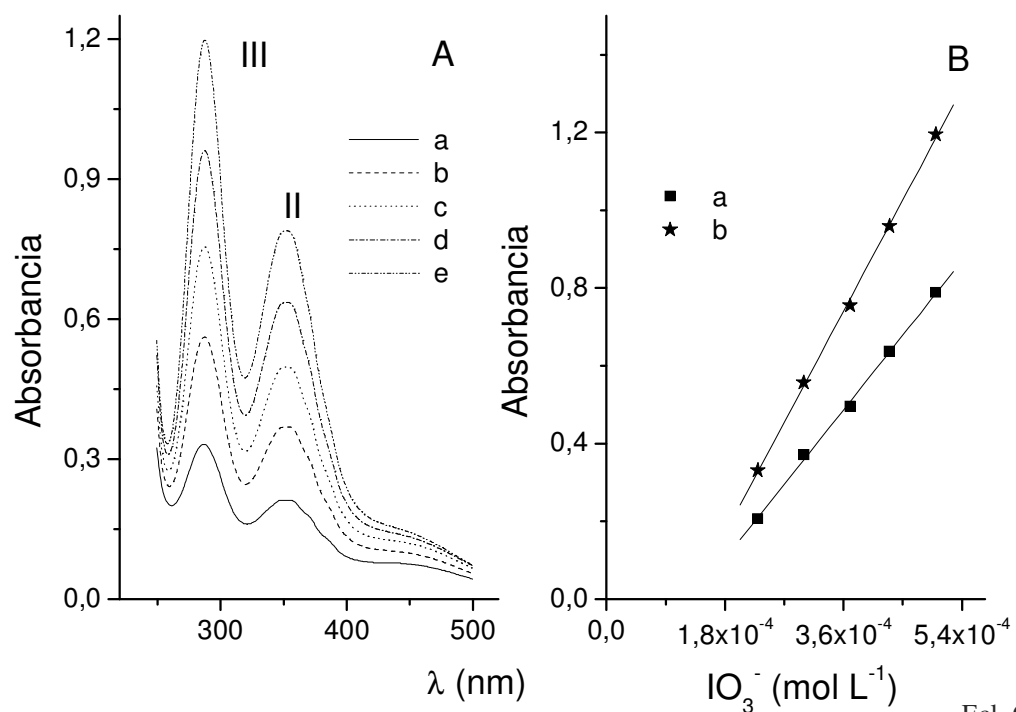

Ecl. Quím., São Paulo, 36 ,2011 105 
Figura 6A: Espectros de absorção UV-Vis obtidos em B-R pH para misturas de: (curva "a") $2,3 \times 10^{-4} \mathrm{~mol} \mathrm{~L}^{-1}$ de $\mathrm{IO}_{3}^{-}$e $7,0 \times 10^{-4} \mathrm{~mol} \mathrm{~L}^{-1}$ de $\mathrm{NH}_{2} \mathrm{OH}$; (curva "b") 3,0x $10^{-4} \mathrm{~mol} \mathrm{~L}^{-1}$ de $\mathrm{IO}_{3}{ }^{-} \mathrm{e}$ 9,0x $10^{-4} \mathrm{~mol} \mathrm{~L}^{-1}$ de $\mathrm{NH}_{2} \mathrm{OH}$; (curva "c") $3,7 \times 10^{-4} \mathrm{~mol} \mathrm{~L}^{-1}$ de $\mathrm{IO}_{3}{ }^{-}$e $1,1 \times 10^{-3} \mathrm{~mol} \mathrm{~L}^{-1}$ de $\mathrm{NH}_{2} \mathrm{OH}$;

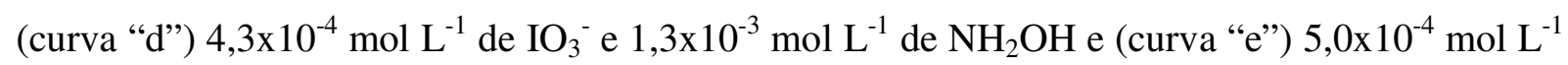
de $\mathrm{IO}_{3}{ }^{-}$e $1,5 \times 10^{-3} \mathrm{~mol} \mathrm{~L}^{-1}$ de $\mathrm{NH}_{2} \mathrm{OH}$. Figura 6B: Curvas analíticas obtidas de 6A. Curva "a" pico II e "b" pico III.

As curvas espectrais da Figura 6A mostram que os sinais dos picos I e II aumentam em função do aumento da concentração de $\mathrm{IO}_{3}{ }^{-}$. Todavia, estes resultados foram obtidos quando se mantém constante a relação de 1:3 entre as concentrações de iodato e hidroxilamina, ou seja, também é necessário o incremento constante de $\mathrm{NH}_{2} \mathrm{OH}$ nos aumentos da concentração de $\mathrm{IO}_{3}{ }^{-}$. Duas curvas retilíneas (Figura 6B) foram obtidas a partir dos espectros mostrados em 6A. As curvas podem ser representadas pelas equações: (pico II), $\mathrm{Y}=-0,28+2,13 \times 10^{3}\left[\mathrm{IO}_{3}{ }^{-}\right], \mathrm{R}=0,9992, \mathrm{n}=5 \mathrm{e}$ (pico III), $\mathrm{Y}=-0,40+3,18 \times 10^{3}\left[\mathrm{IO}_{3}{ }^{-}\right], \mathrm{R}=0,9995, \mathrm{n}=5$, onde $\left[\mathrm{IO}_{3}{ }^{-}\right]$é a concentração de iodado $\left(m o l L^{-1}\right)$, "R" o coeficiente de correlação e "n" o número de medidas. A avaliação destes dados indica que os sinais observados para o pico III $(\lambda=288 \mathrm{~nm})$ apresentam maior sensibilidade analítica em comparação com o pico II $(\lambda=352 \mathrm{~nm})$.

A aplicação da metodologia desenvolvida foi realizada em amostras comerciais, facilmente encontradas nos supermercados da cidade de Natal-RN. As marcas selecionadas foram os sais: Marlin, Nevado e Leste. Os resultados obtidos para cada amostra são mostrados na Tabela 1.

Tabela 1: Valores de iodato encontrados nas amostras comerciais selecionadas.

\begin{tabular}{|l|l|l|}
\hline Amostras & $\begin{array}{l}\text { Iodato encontrado } \\
\left(\mathrm{mg} \mathrm{Kg}^{-1}\right)\end{array}$ & $\begin{array}{l}\text { Desvio-padrão * } \\
( \pm)\end{array}$ \\
\hline Marlin & 40 & 0,5 \\
\hline Nevado & 42 & 0,8 \\
\hline Leste & 45 & 0,2 \\
\hline
\end{tabular}

(*) para 3 medidas. 
De acordo com a ANVISA [45], somente será considerado próprio para consumo humano, o sal que contiver teor igual ou superior a 20 (vinte) miligramas, até o limite máximo de 60 (sessenta) miligramas de iodo por quilograma de produto. Os resultados encontrados, para iodato de potássio nas matrizes analisadas, apresentaram valores que atendem as exigências da legislação especifica. Todo o tratamento estatístico foi realizado com o auxilio da literatura $[46,47]$.

\section{Conclusões}

A redução de iodato, na presença de excesso de hidroxilamina (1:3) em meio ácido, promove a formação do complexo triiodeto, o qual pode ser monitorado espectrofotometricamente através de duas bandas de absorção na região do ultravioleta. Utilizando as condições ótimas de trabalho, foi possível a construção de duas curvas analíticas empregando os picos em $\lambda_{\max } 352 \mathrm{~nm}$ e $\lambda_{\max }$. $285 \mathrm{~nm}$. A aplicação da metodologia em amostras comerciais permitiu a determinação de iodato em sais de cozinha e, a comparação dos valores obtidos com àqueles discriminados na legislação vigente, mostrou que o sistema proposto é viável para a análise quantitativa de iodato.

\section{Referências}

[1] R. L. G. N. P. Silva, A. F. Oliveira, E. A. Neves, J. Braz. Chem. Soc., 9(2) (1998) 171.

[2] H. S. Shin, Y. S. O. Shin, J. H. Kim, J. K. Ryu, Journal of Chromatography A, 732 (1996) 327.

[3] E. Aitken, Journal of the New Zealand Dietetic Association, 55 (2001) 4.

[4] K. L. Hynes, C. L. Blizzard, A. J. Venn, T. Dwyer, J. R. Burgess, Journal of Public Health, 28 (2004) 476.

[5] L. Dahl, L. Johansson, K. Julshamm, H. M. Meltzer, Public Health Nutrition, 7 (2003) 569.

[6] S. Katamine, Y. Mamiya, K. Sekimoto, N. Hoshino, K. Totsuka, M. Suzuki, Nutrition Reports International, 35 (1987) 289.

[7] M. B. Zimmermann, C. Zeder, N. Chaouki, A. Saad, T. Torresani, R. F. Hurrell, American Journal of Clinical Nutrition, 77 (2003) 425.

[8] E. J. Kuhajek, H. W. Fiedelman, Food Technology., 27 (1973) 52.

[9] H. Bürgi, T. Schaffner, J. P. Seiler, Thyroid, 11(5) (2001) 449.

[10] D. V. Gonzales, G. C. R. Tallo, M. F. Garcia, Analysis, 106 (1981) 1224. 
[11] M. Barzegar, H. Khajehsharifi, M. F. Mousavi, Can. J. Anal. Sci. Spect., 48 (2003) 303.

[12] A. Afkhami, F. Mosaed, Anal Sci., 18 (2002) 667.

[13] W. X. Ma, Spectrosc. Spect. Anal. 19 (1999) 763.

[14] S. Gosain, M. Pandey, K. K. Verma, AOAC. Int., 82 (1999) 167.

[15] A. A. Ensafi, G. B. Dehaghi, Anal. Sci., 16 (2000) 61.

[16] L. Perring, M. B. Dvorzak, D. Andrey, Analyst, 126 (2001) 985.

[17] A. Afkhami, T. Madrakian, M. Bahram, Journal of Hazardous Materials B, 123 (2005) 250.

[18] A. Afkhami, A. R. Zarei, Talanta, 60 (2003) 63.

[19] A. Afkhami, A. R. Zarei, Talanta, 53 (2001) 815.

[20] M. S. E. Shahawi, F. A. A. Hashemi, Talanta, 43 (1996) 2037.

[21] G. Mary, N. Balasubramanian, K. S. Nagaraja, Chem. Pharm. Bull., 56(7) (2008) 888.

[22] N. Choengchan, K. Uraisin, K. Choden, W. Veerasaia, K. Grudpan, D. Nacaprich, Talanta, 58 (2002) 1195.

[23] J. Jakmunee, K. Grudpan, Anal. Chim. Acta, 438 (2001) 299.

[24] L. Kosminsky, M. Bertotti, Electroanal., 11 (1999) 623.

[25] J. Sun, X. Chen, Z. Hu, Fresenius J. Anal. Chem. 357 (1997) 1002.

[26] A. A. Ensafi, M. A. Chamjangali, Spectrochimica Acta Part A, 59 (2003) 2897.

[27] A. A. Ensafi, M. A. Chamjangali, Spectrochimica Acta Part A, 58 (2002) 2835.

[28] S. D. Kumar, B. Maiti, P. K. Mathur, Talanta, 53 (2001) 701.

[29] H. T. Fu, L. M. Zhao, Y. L. Zhang, Chin. J. Anal. Chem., 27 (1999) 684.

[30] D. P. Gupta, M. A. Jain, K. K. Verma, J. Chromatogr. A, 1023 (2004) 33.

[31] X. R. Xu, H. B. Li, J. D. Gu, K. J. Paeng, Chromatographia, 60(11/12) (2004) 721.

[32] I. Svancara, B. Ogorevc, M. Novic, K. Vytras, Anal. Bioanal. Chem., 372 (2002) 795.

[33] J. Lin, Fresenius J. Anal. Chem. 363 (1999) 706.

[34] E. Marafon, L. T. Kubota, Y. Gushikem, J. Solid. State Electrochem., 13 (2009) 377.

[35] V. Zic, V. W. Truesdale, N. Cukrov, Marine Chemistry, 112 (2008) 168.

[36] X. Huang, Y. Li, Y., Chen, L. Wang, Sensors and Actuators B, 134 (2008) 780.

[37] A. Salimi, B. Kavosi, A. Babaei, R. Hallaj, Analytica Chimica Acta, 618 (2008) 43.

[38] O. V. Zui, A. V. Terletskaya, Fresenius J. Anal. Chem., 351 (1995) 212.

[39] Z. Xie, J. Zhao, Talanta, 63 (2004) 339.

[40] A. Vogel, Química analítica qualitativa, Mestre Jou, São Paulo, SP, 1981. 
[41] G. C. M. Bourke, J. Stedman, J. Chem. Research (S) (1999) 684.

[42] V. Manivannan, R. Tichy, J. B. Goodenough, Journal of Solid State Chemistry, 147 (1999) 269.

[43] C. C. D. Coumes, S. Vargas, D. J. Chopin, F. Devisme, International Journal Chemical Kinetics, 30(11) (1998) 785.

[44] J. D. Lee, Química Inorgânica, $3^{a}$ edição, Edgard Blücher Ltda, São Paulo, SP, 1980, cap. 5 .

[45] Agência Nacional de Vigilância Sanitária (ANVISA), Resolução RDC n ${ }^{\circ}$ 130, de 26/05/2003, Diário Oficial da União (DOU) de 28/05/2003.

[46] D. A. Skoog, D. M. West, F. J. Holler, Fundamentals of Analytical Chemistry, 7ed., Ed. Harcourt College Publishing, 1997.

[47] D. C. Harris, Análise Química Quantitativa, LTC editora, Rio de Janeiro RJ, 2001.

\section{IODATE ANALYSIS IN TABLE SALT}

\section{Abstract}

Iodate can be reduced in solution buffer B-R pH 2,0 by means of hydroxylamine. Depending on the concentration of the reducing agent, it is possible to generate molecular iodine or the triiodide complex. Iodine presents a set of three absorption peaks, whereas the complex only two in the ultraviolet region. Using hydroxylamine excess it is possible to monitor the triiodide in $\lambda_{\max } 352 \mathrm{~nm}$ e $\lambda_{\max } .288 \mathrm{~nm}$. Employing the best experimental conditions, linear calibration curves between $2,3 \times 10^{-4} \mathrm{~mol} \mathrm{~L}^{-1}$ to $5,0 \times 10^{-4} \mathrm{~mol} \mathrm{~L}^{-1}$ were obtained for the determination of iodate. The methodology was applied in table salt samples from supermarkets of the city of Natal, RN.

Keywords: iodate, triiodide, hydroxylamine. 